\title{
High Order Random Walks: Beyond Spectral Gap
}

\author{
Tali Kaufman ${ }^{1}$ \\ Department of Computer Science, Bar-Ilan University, Ramat Gan, Israel \\ kaufmant@mit.edu
}

\section{Izhar Oppenheim}

Department of Mathematics, Ben-Gurion University of the Negev, P.O. Box 653, Be'er-Sheva, Israel

izharo@bgu.ac.il

\begin{abstract}
We study high order random walks on high dimensional expanders on simplicial complexes (i.e., hypergraphs). These walks walk from a $k$-face (i.e., a $k$-hyperedge) to a $k$-face if they are both contained in a $k+1$-face (i.e, a $k+1$ hyperedge). This naturally generalizes the random walks on graphs that walk from a vertex (0-face) to a vertex if they are both contained in an edge (1-face).

Recent works have studied the spectrum of high order walks operators and deduced fast mixing. However, the spectral gap of high order walks operators is inherently small, due to natural obstructions (called coboundaries) that do not happen for walks on expander graphs.

In this work we go beyond spectral gap, and relate the expansion of a function on $k$-faces (called $k$-cochain, for $k=0$, this is a function on vertices) to its structure.

We show a Decomposition Theorem: For every $k$-cochain defined on high dimensional expander, there exists a decomposition of the cochain into $i$-cochains such that the square norm of the $k$-cochain is a sum of the square norms of the $i$-chains and such that the more weight the $k$-cochain has on higher levels of the decomposition the better is its expansion, or equivalently, the better is its shrinkage by the high order random walk operator.
\end{abstract}

The following corollaries are implied by the Decomposition Theorem:

- We characterize highly expanding $k$-cochains as those whose mass is concentrated on the highest levels of the decomposition that we construct. For example, a function on edges (i.e. a 1-cochain) which is locally thin (i.e. it contains few edges through every vertex) is highly expanding, while a function on edges that contains all edges through a single vertex is not highly expanding.

- We get optimal mixing for high order random walks on Ramanujan complexes. Ramanujan complexes are recently discovered bounded degree high dimensional expanders. The optimality in their mixing that we prove here, enable us to get from them more efficient Two-Layer-Samplers than those presented by the previous work of Dinur and Kaufman.

2012 ACM Subject Classification Theory of computation $\rightarrow$ Random walks and Markov chains, Mathematics of computing $\rightarrow$ Spectra of graphs, Mathematics of computing $\rightarrow$ Hypergraphs

Keywords and phrases High Dimensional Expanders, Simplicial Complexes, Random Walk

Digital Object Identifier 10.4230/LIPIcs.APPROX-RANDOM.2018.47

Related Version https://arxiv.org/abs/1707.02799

1 This work was partially funded by ERC grant no. 336283 and BSF grant no. 2012256

(c) (i) Tali Kaufman and Izhar Oppenheim;

cc. licensed under Creative Commons License CC-BY

Approximation, Randomization, and Combinatorial Optimization. Algorithms and Techniques (APPROX/RANDOM 2018).

Editors: Eric Blais, Klaus Jansen, José D. P. Rolim, and David Steurer; Article No. 47; pp. 47:1-47:17

Leibniz International Proceedings in Informatics

LIPICS Schloss Dagstuhl - Leibniz-Zentrum für Informatik, Dagstuhl Publishing, Germany 


\section{Introduction}

In this work we study high order random walks on bounded degree simplicial complexes (i.e., hypergraphs). These walks walk from a $k$-face (i.e., a $k$-hyperedge) to a $k$-face if they are both contained in a $(k+1)$-face (i.e, a $k+1$ hyperedge). This naturally generalizes the random walks on graphs that walk from a vertex (0-face) to a vertex if they are both contained in an edge (1-face). Roughly speaking, we are interested in walks that converge fast to a uniform distribution on the $k$-faces. For obtaining such fast convergence, a necessary condition is that the $k$-faces are connected. Namely, every pair of $k$-faces is connected by a path that is composed of $(k+1)$-faces (e.g., in graph every pair of vertices is connected by a path composed of edges). This high order connectivity is a necessary condition for the high order walk to converge. High order connectivity is hard to achieve in bounded degree complexes (or hypergraphs). For example, a random bounded degree simplicial complex (i.e., a complex chosen with uniform distribution from the set of all complexes with the same number of vertices and the same bound on the degree) does not have the high order connectivity property. In fact, there are only few currently known bounded degree complexes which do have this high order connectivity property. However, high order connectivity only ensures the convergence of high order random walk to a uniform distribution. For fast convergence, we need to require some form of high dimensional expansion. It turns out, that fast mixing of its high order random walk on a simplicial complex can be deduced its links structure. Links are local neighborhoods of faces in the complex (e.g. local neighborhoods of vertices, edges etc). A conclusion of this work, as well as previous works on high order random walks, is that requiring that ALL links (i.e. the local neighborhoods) are expanding implies fast mixing of high order random walks (we will discuss links and their expanding properties in detail momentarily). As said, random bounded degree complexes are not even connected, let alone their links are not good expanders.

In this paper we study high order random walks of simplicial complexes whose links are expanding (we call them local spectral expanders). High order random walks are strongly related to PCP agreement tests; direct product testing and direct sum testing [3]. This relation influenced, in part, the study of high order random walks.

The focus of previous works [8, 3] was to bound the second largest eigenvalue (in an absolute value) of the high order walk operator in complexes whose links are good spectral expanders. Namely, previous works have shown that in complexes with links that are good spectral expanders, every $k$-cochain that is orthogonal to the constant functions is shrinked by the $k$-order random walk operator $M_{k}^{+}$. I.e., by the walk that walk from a $k$-face to a $k$-face through $(k+1)$-face. The shrinkage rate is immediately determined by the second eigenvalue of the walk operator. However, there are natural obstructions (such as coboundaries) that prevents very large spectral gap of the walk operator.

It could well be the case that $k$-cochains with some specific structures are shrinked much better (or equivalently expand much better) than the bound obtained by spectral gap. This is similar in spirit to the small set expansion question in, say, the noisy hypercube [2], where the noisy hypercube is not a good expander so we can not say that general sets expand well; However, methods beyond spectral gap enabled showing that small sets of the noisy hypercube expand very well; this is similar to our goal here.

The focus of this work is to relate the structure of a $k$-cochain $\phi$ to its expansion, or equivalently, to the amount of its shrinkage by the random walk operator $M_{k}^{+}$, in complexes that are local spectral expanders. We provide a decomposition theorem that relates the amount of shrinkage of a $k$-cochain to the structure of the $i$-cochains to which it 
decomposes $0 \leq i \leq k$. Specifically, we decompose $\phi$ into $i$-cochains, $0 \leq i \leq k$, such that $\|\phi\|^{2}=\sum_{i=1}^{k}\left\|\phi_{i}\right\|^{2}$ and show that the more weight $\phi$ has on the top levels the better is its shrinkage by the $k$-order random walk operator.

In particular, we derive the following conclusions from our decomposition theorem:

- We characterize $k$-cochains which do not expand a lot as those whose mass is concentrated on the lower levels of the decomposition that we construct. In particular, we show that "locally thin" binary cochains shrink dramatically by the high order random walk operator on a local spectral high dimensional expander. A binary $k$-cochain is "locally thin" if the degree of the cochain in each $(k-1)$-face is small. For example, a function on edges (i.e. a 1-cochain) which contains few edges through every vertex is locally thin, and hence highly expanding, while a function on edges that contains all edges through a single vertex is not highly expanding.

- We derive an optimal bound on the second eigenvalue (in an absolute value) of the high order random walk operator $M_{k}^{+}$of complexes whose links are good one sided spectral expanders. Recent work of [3] have shown optimal bound on the second eigenvalue (in an absolute value) of $M_{k}^{+}$for complexes whose links are good $\underline{\text { two }}$ sided spectral expanders. Thus, we get optimal bound on high order walks on Ramanujan complexes [9] which are one-sided local-spectral expanders, but NOT two sided spectral expanders. Ramanujan complexes are one of the few currently known examples of bounded degree complexes which are one-sided local-spectral expanders.

Since the links of the well studies Ramanujan complexes are one-sided spectral expanders (but NOT two sided spectral expanders), the result of [3] does not apply to the Ramanujan complexes but only for complexes obtained from Ramanujan complexes (e.g. a $k$-skeleton of $k^{2}$ Ramanujan complex). Our result is the first result obtaining optimal bounds of the spectrum of high order random walks on the Ramanujan complexes themselves. The optimality in the mixing of the Ramanujan complexes that we prove here, enable us to get from them more efficient Two-Layer-Samplers than those presented by [3]. In the following we discuss the notion of two layers sampler (that was introduced by [3]), and discuss the more efficient two-layers-samplers that we get in this work.

\subsection{More efficient Two-Layer-Samplers implied by this work}

The work of [3] that studied agreement expansion and agreement tests introduced to following generalization of a sampler that is called a two-layer-sampler

- Definition 1. An infinite family of tripartite incidence graphs $\left\{G\left(L \cup R \cup W, E_{1} \cup E_{2}\right)\right\}_{l}$ is called a family of two-layer-samplers if there are constants $1 \leq k \leq n, \gamma>0$ such that the following conditions hold:

- $L=[l], R \subseteq\left(\begin{array}{c}{[l]} \\ k\end{array}\right), W \subseteq\left(\begin{array}{c}{[l]} \\ n\end{array}\right)$ such that there is an edge between $r \in R$ and $x \in L$ if $x \subset r$ and such that there is an edge between $w \in W$ and $r \in R$ if $r \subset w$.

- $|R|+|W|=O(l)$ where the constants depend only on $k, n, \gamma$.

- $G$ has the following double expansion property:

$$
(\lambda(G(L, R)))^{2} \leq \frac{1}{k}+\gamma \text { and }(\lambda(G(R, W)))^{2} \leq \frac{k}{n}+\gamma,
$$

where $G(L, R), G(R, W)$ are the respective bipartite graphs and $\lambda$ is the second largest normalized singular value of the appropriate transition matrix. 
The work of [3] has shown that a family of two-layer-samplers as above with sets of size $1, k, n$ could be derived from the $0, k-1, n-1$ faces of a simplicial complexes in which the $n-1$-order random walk has optimal mixing. For obtaining complexes with such optimal mixing of $(n-1)$-order walks [3] used the Ramanujan complexes of dimension $(n-1)^{2}$. Thus, the $1, k, n$-sets that appear in the two-layer samplers that they construct correspond to $0, k-1, n-1$ faces in a Ramanujan complexes of dimension $(n-1)^{2}$. Our result here implies optimal mixing of $(n-1)$-random walks in Ramanujan complexes of dimension $n-1$ themselves (i.e., we do not need to take Ramanujan complexes of dimension $(n-1)^{2}$ in order to argue about optimality of $d-1$-random walks.). Thus, we get a two-layer sampler whose $1, k, n$ sets correspond to $0, k-1, n-1$ faces in a Ramanujan complex of dimension $(n-1)$ (and not dim $\left.(n-1)^{2}\right)$. The fact that we use Ramanujan complexes of smaller dimension implies that our sampler uses sets $L, R, W$ which are smaller than those obtained by [3] and hence our two-layer-samplers are more efficient.

\subsection{On simplicial complexes and localization}

A pure $n$-dimensional simplicial complex $X$ is a simplicial complex in which every simplex is contained in an $n$-dimensional simplex. In other words, it is an $(n+1)$-hypergraph with a closure property: for every hyperedge in the hypergraph, all of its subsets are also hyperedges in the hypergraph. The sets with $i+1$ elements are denoted $X(i), 0 \leq i \leq n$. The one-skeleton of the complex $X$ is its underlying graph obtained by $X(0) \cup X(1)$. A set $\tau \in X(i)$ is called a face. The link of $\tau$ denoted $X_{\tau}$ is the complex obtained by taking all faces in $X$ that contain $\tau$ and removing $\tau$ from them. Thus, if $\tau$ is of dimension $i$ (i.e. $\tau \in X(i))$ then $X_{\tau}$ is of dimension $n-i-1$.

For every $-1 \leq i \leq n-2$, the one skeleton of $X_{\tau}$ is a graph. Its second largest eigenvalue is $\mu_{\tau}$; its smallest eigenvalue is $\nu_{\tau}$.

- Definition 2 (One sided local spectral expander). A pure $n$-dimensional complex $X$ is a one-sided $\lambda$-local-spectral expander if for every $-1 \leq i \leq n-2$, and for every $\tau \in X(i)$, $\mu_{\tau} \leq \lambda$.

- Definition 3 (Two sided local spectral expander). A pure $n$-dimensional complex $X$ is a two-sided $\lambda$-local-spectral expander if for every $-1 \leq i \leq n-2$, and for every $\tau \in X(i)$, $-\lambda \leq \nu_{\tau}$ and $\mu_{\tau} \leq \lambda$

\subsection{A decomposition theorem for high order random walks and its implications}

We study the random walk operators: $M_{k}^{+}$, corresponding to the walk from a $k$-face to a $k$-face through $k+1$ face. (For exact definition see Section 2). We normalize the operator so that the largest eigenvalue is 1 .

In this paper we show the following decomposition theorem that for one-sided $\lambda$-localspectral expanders:

- Theorem 4 (decomposition Theorem, informal, for formal see Theorem 18). Given a pure $n$ dimensional one-sided $\lambda$-local-spectral expander $X$. For a $k$-cochain $\phi(k \leq n-1)$ orthogonal to the constant functions there exist $i$-cochains $\phi_{i}$ for every $0 \leq i \leq k$ such that $\|\phi\|^{2}=$ $\sum_{i=0}^{k}\left\|\phi_{i}\right\|^{2}$ such that

$$
\left\langle M_{k}^{+} \phi, \phi\right\rangle \leq \sum_{i=0}^{k} \frac{k+1-i}{k+2}\left\|\phi_{i}\right\|^{2}+(k+1) \lambda\|\phi\|^{2} .
$$


As a corollary of the decomposition theorem we derive optimal bounds on the second largest eigenvalue (in an absolute value) of $M_{k}^{+}$for $X$ which is one-sided $\lambda$-local-spectral expander. This result is stronger than [3] that applies only for two-sided $\lambda$-local-spectral expander.

Theorem 5 (Bounding the second eigenvalue of the $k$-walk Theorem, informal, for formal see Theorem 20). Given a pure $n$-dimensional one-sided $\lambda$-local-spectral expander $X$. For a $k$-cochain $\phi(k \leq n-1)$ orthogonal to the constant functions:

$$
\left\|M_{k}^{+} \phi\right\| \leq\left(\left(1-\frac{1}{k+2}\right)+(k+1) \lambda\right)\|\phi\| .
$$

This result improves on previous results: in [8] a similar result is given, but the bound on $\left\|M_{k}^{+} \phi\right\|$ is less tight (the bound is $\left(\left(1-\frac{1}{(k+2)^{2}}\right)+O((k+1) \lambda)\|\phi\|\right)$. In [3], a similar bound is given, but under the stronger assumption that $X$ is a two-sided $\lambda$-local-spectral expander. The seemingly mild improvement that [3] achieves over [8] is crucial for their application. However, as the Ramanujan complexes are only one-sided $\lambda$-local-spectral expanders, the result of [3] does not apply to the Ramanujan complexes themselves but only to other complexes that could be built based on them.

All of the results discussed before are proven with respect to the lazy random walk operator $M_{k}^{+}$. The operator is called lazy since when the walk is located on a certain $k$-face is has non-zero probability that the next step will stay on the same $k$-face and will not move. Similarly one can define a a non-lazy random walk operator (see Definition 8). We show that for complexes that are two-sided $\lambda$-local-spectral expanders, similar results that we obtained for the lazy ransom walks could be obtained also for the non-lazy random walk.

\subsection{On small set expansion phenomenon, the Grassmann complex and our work}

As we have explained above, we study the amount of shrinkage of a $k$-cochain by the random walk operator $M_{k}^{+}$. Our motivation is to go beyond spectral gap and to related the shrinkage of a $k$-cochain by the operator, to its structure. Similar questions are asked in the study of small set expansion in the noisy hypercube [2]. Recently it was shown that studying the structure of non expanding $k$-cochains of the Grassmann complex is strongly related to the "2-to-1 games Conjecture" [4, 5], which is a weaker form of the famous Unique Game Conjecture. Our work here, is of the same flavor. However, instead of working with a specific complex (e.g the Grassmann) we work with simplicial complexes, whose links are good spectral expanders. We characterise non expanding $k$-cochains as those whose mass is concentrated on the lower levels of the decomposition that we construct.

\section{Definitions and notations}

Let $X$ be a pure $n$-dimensional finite simplicial complex. For $-1 \leq k \leq n$, we denote $X(k)$ to be the set of all $k$-simplices in $X(X(-1)=\{\emptyset\})$. A weight function $m$ on $X$ is a function: $m: \bigcup_{-1 \leq k \leq n} X(k) \rightarrow \mathbb{R}^{+}$, such that for every $-1 \leq k \leq n-1$ and for every $\tau \in X(k)$ we have that $m(\tau)=\sum_{\sigma \in X(k+1)} m(\sigma)$. By this definition, it is clear the $m$ is determined by the values in takes in $X(n)$. A simplicial complex with a weight function will be called a weighted simplcial complex. For a pure $n$-dimensional simplicial complex there is a natural weight function $m_{h}$ which we call the homogeneous weight function (since it give the value 1 
to each $n$-dimensional simplex) defined as follows:

$$
\forall \tau \in X(k), m_{h}(\tau)=(n-k) !|\{\eta \in X(n): \tau \subseteq \eta\}| .
$$

We leave it for the reader to verify that $m_{h}$ is indeed a weight function.

Throughout this article, $X$ is a pure $n$-dimensional finite weighted simplicial complex with a weight function $m$.

For $-1 \leq k \leq n-1$, we denote $C^{k}(X, \mathbb{R})$ to be the set of all functions $\phi: X(k) \rightarrow \mathbb{R}$. Abusing the terminology, we will call the space $C^{k}(X, \mathbb{R})$ the space of non-oriented cochains. On $C^{k}(X, \mathbb{R})$ define the following inner-product:

$$
\forall \phi, \psi \in C^{k}(X, \mathbb{R}),\langle\phi, \psi\rangle=\sum_{\sigma \in X(k)} m(\sigma) \phi(\sigma) \psi(\sigma) .
$$

Denote by $\|$.$\| the norm induced by this inner-product.$

\section{Upper and lower random walks}

Let $X$ be a pure $n$-dimensional finite weighted simplicial complex with a weight function $m$. We will define the following random walks on simplices of $X$ :

- Definition 6. For $0 \leq k \leq n-1$, the upper random walk on $k$-simplices is defined by the transition probability matrix $M_{k}^{+}: X(k) \times X(k) \rightarrow \mathbb{R}$ :

$$
M_{k}^{+}\left(\tau, \tau^{\prime}\right)= \begin{cases}\frac{1}{k+2} & \tau=\tau^{\prime} \\ \frac{m\left(\tau \cup \tau^{\prime}\right)}{(k+2) m(\tau)} & \tau \cup \tau^{\prime} \in X(k+1) . \\ 0 & \text { otherwise }\end{cases}
$$

Definition 7. For $0 \leq k \leq n$, the lower random walk on $k$-simplices is defined by the transition probability matrix $M_{k}^{-}: X(k) \times X(k) \rightarrow \mathbb{R}:$

$$
M_{k}^{-}\left(\tau, \tau^{\prime}\right)= \begin{cases}\sum_{\eta \in X(k-1)} \frac{m(\tau)}{(k+1) m(\eta)} & \tau=\tau^{\prime} \\ \frac{m\left(\tau^{\prime}\right)}{(k+1) m\left(\tau \cap \tau^{\prime}\right)} & \tau \cap \tau^{\prime} \in X(k-1) . \\ 0 & \text { otherwise }\end{cases}
$$

We leave it to the reader to check that those are in fact transition probability matrix, i.e., that for every $\tau, \sum_{\tau^{\prime}} M_{k}^{ \pm}\left(\tau, \tau^{\prime}\right)=1$. We note that both the random walks defined above are lazy in the sense that $M^{ \pm}(\tau, \tau) \neq 0$. In the case of the upper random walk, one can easily define a non lazy random walk as follows:

Definition 8. For $0 \leq k \leq n-1$, the non-lazy upper random walk on $k$-simplices is defined by the transition probability matrix $\left(M^{\prime}\right)_{k}^{+}: X(k) \times X(k) \rightarrow \mathbb{R}$ :

$$
\left(M^{\prime}\right)_{k}^{+}=\frac{k+2}{k+1}\left(M_{k}^{+}-\frac{1}{k+2} I\right)=\frac{k+2}{k+1} M_{k}^{+}-\frac{1}{k+1} I .
$$

It is standard to view $M_{k}^{ \pm},\left(M^{\prime}\right)_{k}^{+}$as averaging operators on $C^{k}(X, \mathbb{R})$ and we will not make the distinction between the transition probability matrix and the averaging operator it induces.

It is worth noting that $M_{0}^{-}$and $\left(M^{\prime}\right)_{0}^{+}$are familiar operators/matrices: $M_{0}^{-}$is a projection on the space of the constant functions (on vertices) with respect to the inner-product defined above, and $\left(M^{\prime}\right)_{0}^{+}$is the weighted (normalized) adjacency matrix of the 1-skeleton of $X$. 


\section{The signless differential}

- Definition 9. For $-1 \leq k \leq n-1$, the signless $k$-differential is an operator $d_{k}: C^{k}(X, \mathbb{R}) \rightarrow$ $C^{k+1}(X, \mathbb{R})$ defined as:

$$
\forall \phi \in C^{k}(X, \mathbb{R}), \forall \sigma \in X(k+1), d_{k} \phi(\sigma)=\sum_{\tau \subset \sigma, \tau \in X(k)} \phi(\tau)
$$

Define $\left(d_{k}\right)^{*}: C^{k+1}(X, \mathbb{R}) \rightarrow C^{k}(X, \mathbb{R})$ to be the adjoint operator to $d_{k}$, i.e., the operator such that for every $\phi \in C^{k}(X, \mathbb{R}), \psi \in C^{k+1}(X, \mathbb{R}),\left\langle d_{k} \phi, \psi\right\rangle=\left\langle\phi,\left(d_{k}\right)^{*} \psi\right\rangle$.

- Remark. We note that the signless differential is not a differential in the usual sense, since $d_{k+1} d_{k} \neq 0$. The name signless differential stems from the fact that this is the operator we will use in lieu of the differential in our setting (note that since our non-oriented cochains are defined without using orientation of simplices, we cannot use the usual differential).

Below, we will usually omit the index of signless differential and its adjoint and just denote $d, d^{*}$ where $k$ will be implicit.

Lemma 10. For $-1 \leq k \leq n-1, d^{*}: C^{k+1}(X, \mathbb{R}) \rightarrow C^{k}(X, \mathbb{R})$ is the operator

$$
\forall \psi \in C^{k+1}(X, \mathbb{R}), \forall \tau \in X(k), d^{*} \psi(\tau)=\sum_{\sigma \in X(k+1), \tau \subset \sigma} \frac{m(\sigma)}{m(\tau)} \psi(\sigma) .
$$

Proof. Let $\phi \in C^{k}(X, \mathbb{R})$ and $\psi \in C^{k+1}(X, \mathbb{R})$. Then

$$
\begin{array}{r}
\langle d \phi, \psi\rangle=\sum_{\sigma \in X(k+1)} m(\sigma) d \phi(\sigma) \psi(\sigma)=\sum_{\sigma \in X(k+1)} m(\sigma) \sum_{\tau \in X(k), \tau \subset \sigma} \phi(\tau) \psi(\sigma)= \\
\sum_{\tau \in X(k)} \phi(\tau) \sum_{\sigma \in X(k+1), \tau \subset \sigma} m(\sigma) \psi(\sigma)= \\
\sum_{\tau \in X(k)} m(\tau) \phi(\tau)\left(\sum_{\sigma \in X(k+1), \tau \subset \sigma} \frac{m(\sigma)}{m(\tau)} \psi(\sigma)\right)=\left\langle\phi, d^{*} \psi\right\rangle .
\end{array}
$$

Corollary 11. For $0 \leq k \leq n-1$ and $\phi \in C^{k}(X, \mathbb{R}), d^{*} d \phi=(k+2) M^{+} \phi$ and $d d^{*} \phi=$ $(k+1) M^{-} \phi$.

Proof. Let $\phi \in C^{k}(X, \mathbb{R})$ and $\tau \in X(k)$, then

$$
\begin{aligned}
d^{*} d \phi(\tau)= & \sum_{\sigma \in X(k+1), \tau \subset \sigma} \frac{m(\sigma)}{m(\tau)} d \phi(\sigma)=\sum_{\sigma \in X(k+1), \tau \subset \sigma} \frac{m(\sigma)}{m(\tau)} \sum_{\tau^{\prime} \in X(k), \tau^{\prime} \subset \sigma} \phi\left(\tau^{\prime}\right)= \\
& \sum_{\sigma \in X(k+1), \tau \subset \sigma} \frac{m(\sigma)}{m(\tau)} \sum_{\tau^{\prime} \in X(k), \tau^{\prime} \subset \sigma, \tau^{\prime} \neq \tau} \phi\left(\tau^{\prime}\right)+\sum_{\sigma \in X(k+1), \tau \subset \sigma} \frac{m(\sigma)}{m(\tau)} \phi(\tau) .
\end{aligned}
$$

Note that

$$
\sum_{\sigma \in X(k+1), \tau \subset \sigma} \frac{m(\sigma)}{m(\tau)} \phi(\tau)=\frac{m(\tau)}{m(\tau)} \phi(\tau)=\phi(\tau) .
$$


Also note that

$$
\begin{array}{r}
\sum_{\sigma \in X(k+1), \tau \subset \sigma} \frac{m(\sigma)}{m(\tau)} \sum_{\tau^{\prime} \in X(k), \tau^{\prime} \subset \sigma, \tau^{\prime} \neq \tau} \phi\left(\tau^{\prime}\right)= \\
\sum_{\sigma \in X(k+1), \tau \subset \sigma} \sum_{\tau^{\prime} \in X(k), \tau^{\prime} \subset \sigma, \tau^{\prime} \neq \tau} \frac{m\left(\tau^{\prime} \cup \tau\right)}{m(\tau)} \phi\left(\tau^{\prime}\right)=\sum_{\tau^{\prime} \in X(k), \tau \cup \tau^{\prime} \in X(k+1)} \frac{m\left(\tau \cup \tau^{\prime}\right)}{m(\tau)} \phi\left(\tau^{\prime}\right) .
\end{array}
$$

Therefore

$$
d^{*} d \phi(\tau)=\phi(\tau)+\sum_{\tau^{\prime} \in X(k), \tau \cup \tau^{\prime} \in X(k+1)} \frac{m\left(\tau \cup \tau^{\prime}\right)}{m(\tau)} \phi\left(\tau^{\prime}\right)=(k+2) M^{+} \phi(\tau) .
$$

Similarly,

$$
\begin{array}{r}
d d^{*} \phi(\tau)=\sum_{\eta \in X(k-1), \eta \subset \tau} d^{*} \phi(\eta)=\sum_{\eta \in X(k-1), \eta \subset \tau} \sum_{\sum^{\prime} \in X(k), \eta \subset \tau^{\prime}} \frac{m\left(\tau^{\prime}\right)}{m(\eta)} \phi\left(\tau^{\prime}\right)= \\
\sum_{\eta \in X(k-1), \eta \subset \tau \tau^{\prime} \in X(k), \tau^{\prime} \neq \tau, \eta \subset \tau^{\prime}} \frac{m\left(\tau^{\prime}\right)}{m(\eta)} \phi\left(\tau^{\prime}\right)+\sum_{\eta \in X(k-1), \eta \subset \tau} \frac{m(\tau)}{m(\eta)} \phi(\tau)= \\
\sum_{\eta \in X(k-1), \eta \subset \tau \tau^{\prime} \in X(k), \tau^{\prime} \cap \tau=\eta} \frac{m\left(\tau^{\prime}\right)}{m\left(\tau \cap \tau^{\prime}\right)} \phi\left(\tau^{\prime}\right)+\sum_{\eta \in X(k-1), \eta \subset \tau} \frac{m(\tau)}{m(\eta)} \phi(\tau)= \\
\frac{m\left(\tau^{\prime}\right)}{m\left(\tau \cap \tau^{\prime}\right)} \phi\left(\tau^{\prime}\right)+\sum_{\eta \in X(k-1), \eta \subset \tau} \frac{m(\tau)}{m(\eta)} \phi(\tau)=(k+1) M^{-} \phi(\tau) .
\end{array}
$$

\section{$5 \quad$ Links and localization}

Let $X$ be a pure $n$-dimensional finite simplicial complex with a weight function $m$. Recall that for $-1 \leq k \leq n-1, \tau \in X(k)$, the link of $\tau$, denoted $X_{\tau}$, is a pure $(n-k-1)$-simplicial complex defined as:

$\eta \in X_{\tau}(l) \Leftrightarrow \eta \in X(l)$ and $\tau \cup \eta \in X(k+l+1)$.

On $X_{\tau}$ we define the weight function $m_{\tau}$ induced by $m$ as

$$
m_{\tau}(\eta)=m(\tau \cup \eta)
$$

Using this weight function the inner-product and the norm on $C^{l}\left(X_{\tau}, \mathbb{R}\right)$ are defined as above. The operators $M_{\tau, l}^{ \pm},\left(M^{\prime}\right)_{\tau, l}^{+}$and $d_{\tau}, d_{\tau}^{*}$ are also defined on $C^{l}\left(X_{\tau}, \mathbb{R}\right)$ as above.

Given a cochain $\phi \in C^{l}(X, \mathbb{R})$ and a simplex $\tau \in X(k)$ with $-1 \leq k<l$, we define the localization of $\phi$ on $X_{\tau}$, denoted $\phi_{\tau}$ as a cochain $\phi_{\tau} \in C^{l-k-1}\left(X_{\tau}, \mathbb{R}\right)$ defined as

$$
\phi_{\tau}(\eta)=\phi(\tau \cup \eta)
$$

The key observation (which was initially due to Garland [6], but is now considered standard - see [1], [7]) is that the norm of $\phi, d^{*} \phi$, and $d \phi$ can be calculated via their localizations. Also, the work of the second named author implies that the local spectral information needed to bound $d \phi$ can be deduced from the spectral information of the 1-dimensional links. The proof are somewhat technical and therefore below we will just state the results and give the proofs in the appendix. 
- Proposition 12. Let $-1 \leq k<l \leq n$ and let $\phi \in C^{l}(X, \mathbb{R})$, then

$$
\left(\begin{array}{c}
l+1 \\
k+1
\end{array}\right)\|\phi\|^{2}=\sum_{\tau \in X(k)}\left\|\phi_{\tau}\right\|^{2} \text { and }\left(\begin{array}{c}
l \\
k+1
\end{array}\right)\left\|d^{*} \phi\right\|^{2}=\sum_{\tau \in X(k)}\left\|d_{\tau}^{*} \phi_{\tau}\right\|^{2} .
$$

Also, if $l<n$, then

$$
\|d \phi\|^{2}=\sum_{\tau \in X(l-1)}\left(\left\|d_{\tau} \phi_{\tau}\right\|^{2}-\frac{l}{l+1}\left\|\phi_{\tau}\right\|^{2}\right) .
$$

As a result of Proposition 12 we deduce the following:

- Proposition 13. Let $-1 \leq k \leq n-1$ and let $\phi \in C^{k}(X, \mathbb{R})$, then

$$
\|d \phi\|^{2}=\left\|d^{*} \phi\right\|^{2}+\|\phi\|^{2}+\sum_{\tau \in X(k-1)}\left\langle\left(M^{\prime}\right)_{\tau, 0}^{+}\left(I-M_{\tau, 0}^{-}\right) \phi_{\tau}, \phi_{\tau}\right\rangle .
$$

In light of the above Proposition, we will want to bound the expression

$$
\sum_{\tau \in X(k-1)}\left\langle\left(M^{\prime}\right)_{\tau, 0}^{+}\left(I-M_{\tau, 0}^{-}\right) \phi_{\tau}, \phi_{\tau}\right\rangle,
$$

using spectral information about $X$. To make this precise, we will recall/define the following. For $0 \leq k \leq n-1$ and $\tau \in X(k-1)$, recall that by Corollary $11,\left(M^{\prime}\right)_{\tau, 0}^{+}=d_{\tau}^{*} d_{\tau}-I$ and therefore the eigenvalues of $\left(M^{\prime}\right)_{\tau, 0}^{+}$are real. Denote $\mu_{\tau}$ to be the second largest eigenvalue of $\left(M^{\prime}\right)_{\tau, 0}^{+}$and $\nu_{\tau}$ to be the smallest eigenvalue of $\left(M^{\prime}\right)_{\tau, 0}^{+}$. Note that if 1-skeleton of $X_{\tau}$ is connected, then for every eigenfunction $\psi \in C^{0}\left(X_{\tau}, \mathbb{R}\right)$, if $\psi \perp \operatorname{Im} M_{\tau, 0}^{-}$, then $\left(M^{\prime}\right)_{\tau, 0}^{+} \psi=\mu \psi$ with $\nu_{\tau} \leq \mu \leq \mu_{\tau}<1$. Denote

$$
\mu_{k}=\max _{\tau \in X(k-1)} \mu_{\tau}, \nu_{k}=\min _{\tau \in X(k-1)} \nu_{\tau} .
$$

Lemma 14. For every $0 \leq k \leq n-1$ and every $\phi \in C^{k}(X, \mathbb{R})$ we have that

$$
\sum_{\tau \in X(k-1)}\left\langle\left(M^{\prime}\right)_{\tau, 0}^{+}\left(I-M_{\tau, 0}^{-}\right) \phi_{\tau}, \phi_{\tau}\right\rangle \leq(k+1) \mu_{k}\|\phi\|^{2},
$$

and

$$
\sum_{\tau \in X(k-1)}\left\langle\left(M^{\prime}\right)_{\tau, 0}^{+}\left(I-M_{\tau, 0}^{-}\right) \phi_{\tau}, \phi_{\tau}\right\rangle \geq(k+1) \nu_{k}\|\phi\|^{2}
$$

We recall the following definition from the introduction:

- Definition 15 (Local spectral expander). A $n$-dimensional complex $X$ is a one-sided $\lambda$ local-spectral expander if for every $0 \leq k \leq n-1, \mu_{k} \leq \lambda$. A $n$-dimensional complex $X$ is a two-sided $\lambda$-local-spectral expander if for every $0 \leq k \leq n-1,-\lambda \leq \nu_{k}$ and $\mu_{k} \leq \lambda$.

Therefore for one-sided $\lambda$-local-spectral expanders, $\lambda$ can be used to bound $\sum_{\tau \in X(k-1)}\left\langle\left(M^{\prime}\right)_{\tau, 0}^{+}\left(I-M_{\tau, 0}^{-}\right) \phi_{\tau}, \phi_{\tau}\right\rangle$ from above (and from below in the case of two-sided $\lambda$-local-spectral expanders).

We recall the following result appearing in [11][Lemma 5.1] (see also [10][Proposition $3.7])$ :

Lemma 16. Let $X$ be a weighted pure $n$-dimensional simplicial complex, such that all the links of $X$ of dimension $\geq 1$ (including $X$ itself) are connected, then for every $0 \leq k \leq n-2$,

$$
\mu_{k} \leq \frac{\mu_{k+1}}{1-\mu_{k+1}} \text { and } \nu_{k} \geq \frac{\nu_{k+1}}{1-\nu_{k+1}} .
$$




\section{Decomposition theorem for upper random walks}

For every $0 \leq k \leq n-1$, we denote $C_{0}^{k}(X, \mathbb{R})$ to be

$$
C_{0}^{k}(X, \mathbb{R})=\left\{\phi \in C^{k}(X, \mathbb{R}): \sum_{\sigma \in X(k)} m(\sigma) \phi(\sigma)=0\right\}
$$

Let $\mathbb{1}_{k}$ to be the constant 1 function in $C^{k}(X, \mathbb{R})$, then by definition for every $\phi \in C_{0}^{k}(X, \mathbb{R})$, we have that $\left\langle\phi, \mathbb{1}_{k}\right\rangle=\sum_{\sigma \in X(k)} m(\sigma) \phi(\sigma)=0$, and one can see that $C^{k}(X, \mathbb{R})$ has the orthogonal decomposition $C^{k}(X, \mathbb{R})=\operatorname{span}\left\{\mathbb{1}_{k}\right\} \oplus C_{0}^{k}(X, \mathbb{R})$. It is easy to see that $M_{k}^{ \pm} \mathbb{1}_{k}=\mathbb{1}_{k}$ and since, by Corollary $11, M_{k}^{+}, M_{k}^{-}$are self-adjoint operators, is follows that $M_{k}^{ \pm}\left(C_{0}^{k}(X, \mathbb{R})\right) \subseteq$ $C_{0}^{k}(X, \mathbb{R})$.

- Lemma 17. For $0 \leq k \leq n-1, \operatorname{ker}\left(\left(d_{k-1}\right)^{*}\right) \subseteq C_{0}^{k}(X, \mathbb{R})$ and

$$
\forall \psi \in C^{k-1}(X, \mathbb{R}), d_{k-1} \psi \in C_{0}^{k}(X, \mathbb{R}) \Rightarrow \psi \in C_{0}^{k-1}(X, \mathbb{R}) .
$$

Proof. We note that by definition $d_{k-1} \mathbb{1}_{k-1}=(k+1) \mathbb{1}_{k}$, and, by Lemma 10, $\left(d_{k-1}\right)^{*} \mathbb{1}_{k}=$ $\mathbb{1}_{k-1}$. Therefore for every $\phi \in \operatorname{ker}\left(\left(d_{k-1}\right)^{*}\right)$, we have that

$$
0=\left\langle\left(d_{k-1}\right)^{*} \phi, \mathbb{1}_{k-1}\right\rangle=\left\langle\phi,\left(d_{k-1}\right) \mathbb{1}_{k-1}\right\rangle=(k+1)\left\langle\phi,\left(d_{k-1}\right) \mathbb{1}_{k}\right\rangle \Rightarrow \phi \in C_{0}^{k}(X, \mathbb{R}) .
$$

Also, for every $\psi \in C^{k-1}(X, \mathbb{R})$ such that $d_{k-1} \psi \in C_{0}^{k}(X, \mathbb{R})$, we have that

$$
0=\left\langle d_{k-1} \psi, \mathbb{1}_{k}\right\rangle=\left\langle\psi,\left(d_{k-1}\right)^{*} \mathbb{1}_{k}\right\rangle=\left\langle\psi, \mathbb{1}_{k-1}\right\rangle \Rightarrow \psi \in C_{0}^{k-1}(X, \mathbb{R}) .
$$

- Theorem 18 (Decomposition Theorem). For every $0 \leq k \leq n-1$ and every $\phi \in C_{0}^{k}(X, \mathbb{R})$, there are $\phi^{k} \in C_{0}^{k}(X, \mathbb{R}), \phi^{k-1},\left(\phi^{k-1}\right)^{\prime} \in C_{0}^{k-1}(X, \mathbb{R}), \ldots, \phi^{0},\left(\phi^{0}\right)^{\prime} \in C_{0}^{0}(X, \mathbb{R})$ such that if we denote $\left(\phi^{k}\right)^{\prime}=\phi$, then the following holds:

1. For every $0 \leq i \leq k,\left\|\left(\phi^{i}\right)^{\prime}\right\|^{2}=\left\|\phi^{i}\right\|^{2}+\left\|\phi^{i-1}\right\|^{2}+\ldots+\left\|\phi^{0}\right\|^{2}$.

2. $\|d \phi\|^{2}=\sum_{i=0}^{k}(k+1-i)\left\|\phi^{i}\right\|^{2}+\sum_{i=0}^{k} \sum_{\tau \in X(i-1)}\left\langle\left(M^{\prime}\right)_{\tau, 0}^{+}\left(I-M_{\tau, 0}^{-}\right)\left(\phi^{i}\right)_{\tau}^{\prime},\left(\phi^{i}\right)_{\tau}^{\prime}\right\rangle$.

Proof. We will prove the theorem by induction on $k$. For $k=0$ and $\phi \in C_{0}^{0}(X, \mathbb{R})$, we take $\phi^{0}=\phi$ and check that the theorem holds for this choice.

1. This condition holds trivially.

2. We note that $\phi \in C_{0}^{0}(X, \mathbb{R})$ implies that $d^{*} \phi=0$ and therefore this condition follows from Proposition 13.

Assume next that $k>0$ and that the theorem holds for $k-1$. For $\phi \in C_{0}^{k}(X, \mathbb{R})$, we first decompose $\phi$ as $\phi=\phi^{k}+\phi^{\prime}$, where $\phi^{k} \in \operatorname{ker}\left(\left(d_{k-1}\right)^{*}\right)$ and $\left.\phi^{\prime} \in\left(\operatorname{ker}\left(d_{k-1}\right)^{*}\right)\right)^{\perp}$. This is an orthogonal decomposition and therefore $\|\phi\|^{2}=\left\|\phi^{k}\right\|^{2}+\left\|\phi^{\prime}\right\|^{2}$. Also, by Proposition 13,

$$
\left\|d_{k} \phi\right\|^{2}=\|\phi\|^{2}+\left\|\left(d_{k-1}\right)^{*} \phi^{\prime}\right\|^{2}+\sum_{\tau \in X(k-1)}\left\langle\left(M^{\prime}\right)_{\tau, 0}^{+}\left(I-M_{\tau, 0}^{-}\right) \phi_{\tau}, \phi_{\tau}\right\rangle .
$$

We note that $\left.\left(\operatorname{ker}\left(d_{k-1}\right)^{*}\right)\right)^{\perp}=\operatorname{Im}\left(d_{k-1}\right)$ and therefore, by using Lemma 17, there is $\psi \in C_{0}^{k-1}(X, \mathbb{R})$ such that $d_{k-1} \psi=\phi^{\prime}$. This yields that there is $\psi \in C_{0}^{k-1}(X, \mathbb{R})$, such that $\left\|d_{k-1} \psi\right\|^{2}=\left\|\phi^{\prime}\right\|^{2}$ and

$$
\|d \phi\|^{2}=\|\phi\|^{2}+\left\|\left(d_{k-1}\right)^{*} d_{k-1} \psi\right\|^{2}+\sum_{\tau \in X(i-1)}\left\langle\left(M^{\prime}\right)_{\tau, 0}^{+}\left(I-M_{\tau, 0}^{-}\right) \phi_{\tau}, \phi_{\tau}\right\rangle .
$$

We recall that since $\left(d_{k-1}\right)^{*} d_{k-1}$ is a self-adjoint operator, with non negative eigenvalues, $\sqrt{\left(d_{k-1}\right)^{*} d_{k-1}}$ is the self-adjoint operator, with non negative eigenvalues defined as follows: 
for every eigenfunction $\varphi$ of $\left(d_{k-1}\right)^{*} d_{k-1}$ with an eigenvalue $\mu, \varphi$ is an eigenfunction of $\sqrt{\left(d_{k-1}\right)^{*} d_{k-1}}$ with the eigenvalue $\sqrt{\mu}$.

We will take $\left(\phi^{k-1}\right)^{\prime}=\sqrt{\left(d_{k-1}\right)^{*} d_{k-1}} \psi$ and check that the theorem holds for this choice.

First, we note that, using corollary $11,\left(d_{k-1}\right)^{*} d_{k-1}\left(C_{0}^{k-1}(X, \mathbb{R})\right) \subseteq C_{0}^{k-1}(X, \mathbb{R})$, and therefore $\sqrt{\left(d_{k-1}\right)^{*} d_{k-1}}\left(C_{0}^{k-1}(X, \mathbb{R})\right) \subseteq C_{0}^{k-1}(X, \mathbb{R})$, which implies that $\left(\phi^{k-1}\right)^{\prime}=\sqrt{\left(d_{k-1}\right)^{*} d_{k-1}} \psi \in C_{0}^{k-1}(X, \mathbb{R})$.

Second, we note that

$$
\begin{aligned}
\left\|d_{k-1} \psi\right\|^{2} & =\left\langle\left(d_{k-1}\right)^{*} d_{k-1} \psi, \psi\right\rangle= \\
\left\langle\sqrt{\left(d_{k-1}\right)^{*} d_{k-1}} \psi, \sqrt{\left(d_{k-1}\right)^{*} d_{k-1}} \psi\right\rangle & =\left\|\sqrt{\left(d_{k-1}\right)^{*} d_{k-1}} \psi\right\|^{2} .
\end{aligned}
$$

Therefore, $\sqrt{\left(d_{k-1}\right)^{*} d_{k-1}} \psi \in C_{0}^{k-1}(X, \mathbb{R})$ and $\left\|\sqrt{\left(d_{k-1}\right)^{*} d_{k-1}} \psi\right\|=\left\|\phi^{\prime}\right\|$. This yields that

$$
\|\phi\|^{2}=\left\|\phi^{k}\right\|^{2}+\left\|\left(\phi^{k-1}\right)^{\prime}\right\|^{2},
$$

and by the induction assumption

$$
\|\phi\|^{2}=\left\|\phi^{k}\right\|^{2}+\left\|\phi^{k-1}\right\|^{2}+\ldots+\left\|\phi^{0}\right\|^{2} .
$$

Last, we note that

$$
\begin{array}{r}
\left\|\left(d_{k-1}\right)^{*} \phi^{\prime}\right\|^{2}=\left\|\left(d_{k-1}\right)^{*} d_{k-1} \psi\right\|^{2}=\left\langle\left(d_{k-1}\right)^{*} d_{k-1} \psi,\left(d_{k-1}\right)^{*} d_{k-1} \psi\right\rangle= \\
\left\langle\left(d_{k-1}\right)^{*} d_{k-1} \sqrt{\left(d_{k-1}\right)^{*} d_{k-1}} \psi, \sqrt{\left(d_{k-1}\right)^{*} d_{k-1}} \psi\right\rangle=\left\|d_{k-1} \sqrt{\left(d_{k-1}\right)^{*} d_{k-1}} \psi\right\|^{2}= \\
\left\|d_{k-1}\left(\phi^{k-1}\right)^{\prime}\right\|^{2} .
\end{array}
$$

Combining this with (1), we get that

$$
\|d \phi\|^{2}=\|\phi\|^{2}+\left\|d_{k-1}\left(\phi^{k-1}\right)^{\prime}\right\|^{2}+\sum_{\tau \in X(k-1)}\left\langle\left(M^{\prime}\right)_{\tau, 0}^{+}\left(I-M_{\tau, 0}^{-}\right) \phi_{\tau}, \phi_{\tau}\right\rangle .
$$

By the induction assumption,

$$
\left\|d_{k-1}\left(\phi^{k-1}\right)^{\prime}\right\|^{2}=\sum_{i=0}^{k-1}(k-i)\left\|\phi^{i}\right\|^{2}+\sum_{i=0}^{k-1} \sum_{\tau \in X(i-1)}\left\langle\left(M^{\prime}\right)_{\tau, 0}^{+}\left(I-M_{\tau, 0}^{-}\right)\left(\phi^{i}\right)_{\tau}^{\prime},\left(\phi^{i}\right)_{\tau}^{\prime}\right\rangle .
$$

Therefore

$$
\begin{array}{r}
\|d \phi\|^{2}=\|\phi\|^{2}+\left\|d_{k-1}\left(\phi^{k-1}\right)^{\prime}\right\|^{2}+\sum_{\tau \in X(k-1)}\left\langle\left(M^{\prime}\right)_{\tau, 0}^{+}\left(I-M_{\tau, 0}^{-}\right) \phi_{\tau}, \phi_{\tau}\right\rangle= \\
\|\phi\|^{2}+\sum_{i=0}^{k-1}(k-i)\left\|\phi^{i}\right\|^{2}+\sum_{i=0}^{k-1} \sum_{\tau \in X(i-1)}\left\langle\left(M^{\prime}\right)_{\tau, 0}^{+}\left(I-M_{\tau, 0}^{-}\right)\left(\phi^{i}\right)_{\tau}^{\prime},\left(\phi^{i}\right)_{\tau}^{\prime}\right\rangle+ \\
\sum_{\tau \in X(k-1)}\left\langle\left(M^{\prime}\right)_{\tau, 0}^{+}\left(I-M_{\tau, 0}^{-}\right) \phi_{\tau}, \phi_{\tau}\right\rangle= \\
\|\phi\|^{2}+\sum_{i=0}^{k-1}(k-i)\left\|\phi^{i}\right\|^{2}+\sum_{i=0}^{k} \sum_{\tau \in X(i-1)}\left\langle\left(M^{\prime}\right)_{\tau, 0}^{+}\left(I-M_{\tau, 0}^{-}\right)\left(\phi^{i}\right)_{\tau}^{\prime},\left(\phi^{i}\right)_{\tau}^{\prime}\right\rangle= \\
\sum_{i=0}^{k}(k+1-i)\left\|\phi^{i}\right\|^{2}+\sum_{i=0}^{k} \sum_{\tau \in X(i-1)}\left\langle\left(M^{\prime}\right)_{\tau, 0}^{+}\left(I-M_{\tau, 0}^{-}\right)\left(\phi^{i}\right)_{\tau}^{\prime},\left(\phi^{i}\right)_{\tau}^{\prime}\right\rangle,
\end{array}
$$

where the last equality is due to (2). 
- Corollary 19. Let $X$ be a pure $n$-dimensional weighted simplicial complex such that all the links of $X$ of dimension $\geq 1$ are connected (including $X$ itself) and let $0 \leq k \leq n-1$. Then for every $\phi \in C_{0}^{k}(X, \mathbb{R})$, there are $\phi^{k} \in C_{0}^{k}(X, \mathbb{R}), \phi^{k-1} \in C_{0}^{k-1}(X, \mathbb{R}), \ldots, \phi^{0} \in C_{0}^{0}(X, \mathbb{R})$, such that

$$
\|\phi\|^{2}=\left\|\phi^{k}\right\|^{2}+\ldots+\left\|\phi^{0}\right\|^{2}
$$

and

$$
\begin{aligned}
& \|d \phi\|^{2} \leq \sum_{i=0}^{k}\left(k+1-i+\sum_{j=i}^{k}(j+1) \mu_{j}\right)\left\|\phi^{i}\right\|^{2}, \\
& \|d \phi\|^{2} \geq \sum_{i=0}^{k}\left(k+1-i+\sum_{j=i}^{k}(j+1) \nu_{j}\right)\left\|\phi^{i}\right\|^{2} .
\end{aligned}
$$

Proof. Let $\phi \in C_{0}^{k}(X, \mathbb{R})$ and $\phi^{k} \in C_{0}^{k}(X, \mathbb{R}), \phi^{k-1},\left(\phi^{k-1}\right)^{\prime} \in C_{0}^{k-1}(X, \mathbb{R}), \ldots, \phi^{0},\left(\phi^{0}\right)^{\prime} \in$ $C_{0}^{0}(X, \mathbb{R})$ as in the Decomposition Theorem. Then $\|\phi\|^{2}=\left\|\phi^{k}\right\|^{2}+\ldots+\left\|\phi^{0}\right\|^{2}$, and we will prove that $\|d \phi\|^{2} \leq \sum_{i=0}^{k}\left(k+1-i+\sum_{j=i}^{k}(j+1) \mu_{j}\right)\left\|\phi^{i}\right\|^{2}$, (the proof of the second inequality is similar and therefore it is left to the reader).

Note that for every $0 \leq i \leq k$, we have by Lemma 14 that

$$
\sum_{\tau \in X(i-1)}\left\langle\left(M^{\prime}\right)_{\tau, 0}^{+}\left(I-M_{\tau, 0}^{-}\right)\left(\phi^{i}\right)_{\tau}^{\prime},\left(\phi^{i}\right)_{\tau}^{\prime}\right\rangle \leq(i+1) \mu_{i}\left\|\left(\phi^{i}\right)^{\prime}\right\|^{2} .
$$

Therefore

$$
\begin{array}{r}
\sum_{i=0}^{k} \sum_{\tau \in X(i-1)}\left\langle\left(M^{\prime}\right)_{\tau, 0}^{+}\left(I-M_{\tau, 0}^{-}\right)\left(\phi^{i}\right)_{\tau}^{\prime},\left(\phi^{i}\right)_{\tau}^{\prime}\right\rangle \leq \sum_{i=0}^{k}(i+1) \mu_{i} \sum_{j=0}^{i}\left\|\phi^{j}\right\|^{2}= \\
\sum_{j=0}^{k}\left\|\phi^{j}\right\|^{2} \sum_{i=j}^{k}(i+1) \mu_{i} .
\end{array}
$$

Replacing the roles of $i$ and $j$ in the above inequality and combining it with the equation if the Decomposition Theorem for $\|d \phi\|^{2}$ yields the needed inequality.

A consequence of this corollary is the following mixing results for $\lambda$ local spectral expanders:

- Theorem 20 (Mixing of the random walks). Let $X$ be a weighted pure $n$-dimensional simplicial complex and let $0 \leq \lambda \leq 1$ be some constant.

1. If $X$ is a one-sided $\lambda$-local spectral expander, then for every $0 \leq k \leq n-1$,

$$
\forall \phi \in C_{0}^{k}(X, \mathbb{R}),\left\|M_{k}^{+} \phi\right\| \leq\left(\frac{k+1}{k+2}+(k+1) \lambda\right)\|\phi\| .
$$

2. If $X$ is a two-sided $\lambda$-local spectral expander, then for every $0 \leq k \leq n-1$,

$$
\forall \phi \in C_{0}^{k}(X, \mathbb{R}),\left\|\left(M^{\prime}\right)_{k}^{+} \phi\right\| \leq\left(\frac{k}{k+1}+(k+1) \lambda\right)\|\phi\| .
$$




\section{Proof.}

1. Let $0 \leq k \leq n-1$ and $\phi \in C_{0}^{k}(X, \mathbb{R})$. Assume that $X$ is a one-sided $\lambda$-local spectral expander, then by Corollary 19 we get

$$
\begin{array}{r}
\|d \phi\|^{2} \leq \sum_{i=0}^{k}\left(k+1-i+\sum_{j=i}^{k}(j+1) \mu_{j}\right)\left\|\phi^{i}\right\|^{2} \leq \sum_{i=0}^{k}\left(k+1+\sum_{j=0}^{k}(k+1) \lambda\right)\left\|\phi^{i}\right\|^{2}= \\
\sum_{i=0}^{k}\left(k+1+(k+1)^{2} \lambda\right)\left\|\phi^{i}\right\|^{2}=\left(k+1+(k+1)^{2} \lambda\right)\|\phi\|^{2} .
\end{array}
$$

Recall that by corollary $11\left(d_{k}\right)^{*} d_{k}=(k+2) M_{k}^{+}$and therefore

$$
\left\langle M_{k}^{+} \phi, \phi\right\rangle=\frac{1}{k+2}\|d \phi\|^{2} \leq\left(\frac{k+1}{k+2}+\frac{(k+1)^{2}}{k+2} \lambda\right)\|\phi\|^{2} \leq\left(\frac{k+1}{k+2}+(k+1) \lambda\right)\|\phi\|^{2} .
$$

$M_{k}^{+}$is a positive operator that maps $C_{0}^{k}(X, \mathbb{R})$ into itself and therefore, by the above inequality, any eigenvector of $M_{k}^{+}$in $C_{0}^{k}(X, \mathbb{R})$ has an eigenvalue $\leq \frac{k+1}{k+2}+(k+1) \lambda$ so we are done.

2. Let $0 \leq k \leq n-1$ and $\phi \in C_{0}^{k}(X, \mathbb{R})$. Assume that $X$ is a two-sided $\lambda$-local spectral expander. By (3), we have that $\|d \phi\|^{2}-\|\phi\|^{2} \leq\left(k+(k+1)^{2} \lambda\right)\|\phi\|^{2}$. Also, by Corollary 19 , we have that

$$
\begin{aligned}
\|d \phi\|^{2} & \geq \sum_{i=0}^{k}\left(k+1-i+\sum_{j=i}^{k}(j+1) \nu_{j}\right)\left\|\phi^{i}\right\|^{2} \geq \sum_{i=0}^{k}\left(k+1-k+\sum_{j=0}^{k}(k+1)(-\lambda)\right)\left\|\phi^{i}\right\|^{2} \\
& \geq \sum_{i=0}^{k}\left(1-(k+1)^{2} \lambda\right)\left\|\phi^{i}\right\|^{2}=\left(1-(k+1)^{2} \lambda\right)\|\phi\|^{2} .
\end{aligned}
$$

Thus, $\|d \phi\|-\|\phi\|^{2} \geq-(k+1)^{2} \lambda\|\phi\|^{2}$. Note that

$$
\|d \phi\|-\|\phi\|^{2}=\left\langle\left((k+2) M_{+}^{k}-I\right) \phi, \phi\right\rangle=(k+1)\left\langle\left(M^{\prime}\right)_{+}^{k} \phi, \phi\right\rangle .
$$

Therefore, after dividing by $(k+1)$ we showed that

$$
-(k+1) \lambda\|\phi\|^{2} \leq\left\langle\left(M^{\prime}\right)_{+}^{k} \phi, \phi\right\rangle \leq\left(\frac{k}{k+1}+(k+1) \lambda\right)\|\phi\|^{2} .
$$

$\left(M^{\prime}\right)_{+}^{k}$ is an operator with real eigenvalues that maps $C_{0}^{k}(X, \mathbb{R})$ into itself and therefore, by the above inequality, any eigenvector of $M_{k}^{+}$in $C_{0}^{k}(X, \mathbb{R})$ has an eigenvalue between $\frac{k}{k+1}+(k+1) \lambda$ and $-(k+1) \lambda$ so we are done.

Another result of this flavour is mixing theorem for the non-lazy upper random walk in which the condition of the two-sided spectral gap is replaced by the the condition of the one-sided spectral gap and the condition $n>>k$ :

- Theorem 21. Let $X$ be a weighted pure $n$-dimensional simplicial complex and let $0 \leq \lambda \leq 1$ be some constant. If $X$ is a one-sided $\lambda$-local spectral expander, then for every $0 \leq k \leq n-1$,

$$
\forall \phi \in C_{0}^{k}(X, \mathbb{R}),\left\|\left(M^{\prime}\right)_{k}^{+} \phi\right\| \leq\left(\max \left\{\frac{k}{k+1}+(k+1) \lambda, \frac{2(k+1)}{2(n-k-1)-1}\right\}\right)\|\phi\| .
$$

Proof. By Lemma 16, for every $0 \leq i \leq k, \nu_{i} \geq-\frac{1}{n-k}$ and by repeating the same argument as in the proof of the previous theorem completes the proof. 


\section{References}

1 W. Ballmann and J. Świątkowski. On $L^{2}$-cohomology and property (T) for automorphism groups of polyhedral cell complexes. Geom. Funct. Anal., 7(4):615-645, 1997. doi:10. $1007 /$ s000390050022.

2 Boaz Barak, Parikshit Gopalan, Johan Håstad, Raghu Meka, Prasad Raghavendra, and David Steurer. Making the long code shorter. SIAM J. Comput., 44(5):1287-1324, 2015.

3 Irit Dinur and Tali Kaufman. High dimensional expanders imply agreement expanders. Electronic Colloquium on Computational Complexity (ECCC), 2017.

4 Irit Dinur, Subhash Khot, Guy Kindler, Dor Minzer, and Muli Safra. Towards a proof of the 2-to-1 games conjecture? Electronic Colloquium on Computational Complexity (ECCC), 23:198, 2016. URL: http://eccc.hpi-web.de/report/2016/198.

5 Irit Dinur, Subhash Khot, Guy Kindler, Dor Minzer, and Muli Safra. On non-optimally expanding sets in grassmann graphs. Electronic Colloquium on Computational Complexity (ECCC), 24:94, 2017. URL: https://eccc.weizmann.ac.il/report/2017/094.

6 Howard Garland. p-adic curvature and the cohomology of discrete subgroups of $p$-adic groups. Ann. of Math. (2), 97:375-423, 1973.

7 Anna Gundert and Uli Wagner. On Laplacians of random complexes. In Computational geometry (SCG'12), pages 151-160. ACM, New York, 2012. doi:10.1145/2261250.2261272.

8 Tali Kaufman and David Mass. High dimensional combinatorial random walks and colorful expansion. In ITCS, 2017.

9 Alexander Lubotzky, Beth Samuels, and Uzi Vishne. Explicit constructions of ramanujan complexes of type. Eur. J. Comb., 26(6):965-993, 2005. doi:10.1016/j .ejc. 2004.06.007.

10 Izhar Oppenheim. Vanishing of cohomology and property $(\mathrm{T})$ for groups acting on weighted simplicial complexes. Groups Geom. Dyn., 9(1):67-101, 2015. doi:10.4171/GGD/306.

11 Izhar Oppenheim. Local spectral expansion approach to high dimensional expanders Part I: Descent of spectral gaps. Discrete Comput. Geom., 59(2):293-330, 2018. doi:10.1007/ s00454-017-9948-x.

\section{A Proofs of localization results}

Proof of Proposition 12:

Proof. Let $\phi \in C^{l}(X, \mathbb{R})$, then

$$
\begin{array}{r}
\sum_{\tau \in X(k)}\left\|\phi_{\tau}\right\|^{2}=\sum_{\tau \in X(k)} \sum_{\eta \in X_{\tau}^{(l-k-1)}} m_{\tau}(\eta) \phi_{\tau}(\eta)^{2}= \\
\sum_{\tau \in X(k)} \sum_{\eta \in X_{\tau}^{(l-k-1)}} m(\tau \cup \eta) \phi(\tau \cup \eta)^{2}=\sum_{\tau \in X(k)} \sum_{\sigma \in X(l), \tau \subset \sigma} m(\sigma) \phi(\sigma)^{2}= \\
\sum_{\sigma \in X(l)} \sum_{\tau \in X(k), \tau \subset \sigma} m(\sigma) \phi(\sigma)^{2}=\left(\begin{array}{l}
l+1 \\
k+1
\end{array}\right) \sum_{\sigma \in X(l)} m(\sigma) \phi(\sigma)^{2}=\left(\begin{array}{l}
l+1 \\
k+1
\end{array}\right)\|\phi\|^{2} .
\end{array}
$$

In order to prove the second equality, we notice that for every $\tau \in X(k)$ and every $\eta \in$ $X_{\tau}(l-k-2)$, we have that

$$
\begin{array}{r}
\left(d^{*} \phi\right)_{\tau}(\eta)=d^{*} \phi(\tau \cup \eta)=\sum_{\begin{array}{l}
\sigma \in X(l), \tau \cup \eta \subset \sigma \\
\end{array}} \frac{m(\sigma)}{m(\tau \cup \eta)} \phi(\sigma)= \\
\sum_{\sigma \backslash \tau \in X(l-k-1), \eta \subset \sigma \backslash \tau} \frac{m_{\tau}(\sigma \backslash \tau)}{m_{\tau}(\eta)} \phi_{\tau}(\sigma \backslash \tau)=d_{\tau}^{*} \phi_{\tau}(\eta) .
\end{array}
$$


Therefore, $\left(d^{*} \phi\right)_{\tau}=d_{\tau}^{*} \phi_{\tau}$ and by the first equality of this proposition

$$
\left(\begin{array}{c}
l \\
k+1
\end{array}\right)\left\|d^{*} \phi\right\|^{2}=\sum_{\tau \in X(k)}\left\|\left(d^{*} \phi\right)_{\tau}\right\|^{2}=\sum_{\tau \in X(k)}\left\|d_{\tau}^{*} \phi_{\tau}\right\|^{2} .
$$

Assume now that $l<n$, then for every $\sigma \in X(l+1)$, the following holds:

$$
\begin{aligned}
& (d \phi(\sigma))^{2}=\left(\sum_{\eta \in X(l), \eta \subset \sigma} \phi(\eta)\right)^{2}=\sum_{\eta \in X(l), \eta \subset \sigma} \phi(\eta)^{2}+\sum_{\eta, \eta^{\prime} \in X(l), \eta \neq \eta^{\prime}, \eta, \eta^{\prime} \subset \sigma} 2 \phi(\eta) \phi\left(\eta^{\prime}\right)= \\
& \sum_{\eta, \eta^{\prime} \in X(l), \eta \neq \eta^{\prime}, \eta, \eta^{\prime} \subset \sigma}\left(\phi(\eta)+\phi\left(\eta^{\prime}\right)\right)^{2}-l \sum_{\eta \in X(l), \eta \subset \sigma} \phi(\eta)^{2}= \\
& \sum_{\tau \in X(l-1), \tau \subset \sigma}\left(d_{\tau} \phi_{\tau}(\sigma \backslash \tau)\right)^{2}-l \sum_{\eta \in X(l), \eta \subset \sigma} \phi(\eta)^{2} .
\end{aligned}
$$

Therefore

$$
\begin{array}{r}
\|d \phi\|^{2}=\sum_{\sigma \in X(l+1)} m(\sigma)(d \phi(\sigma))^{2}= \\
\sum_{\sigma \in X(l+1)} m(\sigma) \sum_{\tau \in X(l-1), \tau \subset \sigma}\left(d_{\tau} \phi_{\tau}(\sigma \backslash \tau)\right)^{2}-l \sum_{\sigma \in X(l+1)} m(\sigma) \sum_{\eta \in X(l), \eta \subset \sigma} \phi(\eta)^{2}= \\
\sum_{\tau \in X(l-1)} \sum_{\sigma \in X(l+1), \tau \subset \sigma} m(\sigma)\left(d_{\tau} \phi_{\tau}(\sigma \backslash \tau)\right)^{2}-l \sum_{\eta \in X(l)} \phi(\eta)^{2} \sum_{\sigma \in X(l+1), \eta \subset \sigma} m(\sigma)= \\
\sum_{\tau \in X(l-1)} \sum_{\gamma \in X_{\tau}^{(1)}} m_{\tau}(\gamma)\left(d_{\tau} \phi_{\tau}(\gamma)\right)^{2}-l \sum_{\eta \in X(l)} m(\eta) \phi(\eta)^{2}=\sum_{\tau \in X(l-1)}\left\|d_{\tau} \phi_{\tau}\right\|^{2}-l\|\phi\|^{2}= \\
\sum_{\tau \in X(l-1)}\left(\left\|d_{\tau} \phi_{\tau}\right\|^{2}-\frac{l}{l+1}\left\|\phi_{\tau}\right\|^{2}\right),
\end{array}
$$

where the last equality is due to the equality

$$
\|\phi\|^{2}=\frac{1}{l+1} \sum_{\tau \in X(l-1)}\left\|\phi_{\tau}\right\|^{2},
$$

proven above.

Proof of Proposition 13:

Proof. Let $\phi \in C^{k}(X, \mathbb{R})$. Note that for every $\tau \in X(k-1), M_{\tau, 0}^{-}$is the orthogonal projection on the space of constant functions in $C^{0}\left(X_{\tau}, \mathbb{R}\right)$ and therefore $\left(M^{\prime}\right)_{\tau, 0}^{+} M_{\tau, 0}^{-}=M_{\tau, 0}^{-}$.

Further note that by Corollary 11

$$
\begin{array}{r}
\left\|d_{\tau} \phi_{\tau}\right\|^{2}=\left\langle 2 M_{\tau, 0}^{+} \phi_{\tau}, \phi_{\tau}\right\rangle=\left\langle\left(\left(M^{\prime}\right)_{\tau, 0}^{+}+I\right) \phi_{\tau}, \phi_{\tau}\right\rangle= \\
\left\langle\left(M^{\prime}\right)_{\tau, 0}^{+} \phi_{\tau}, \phi_{\tau}\right\rangle+\left\|\phi_{\tau}\right\|^{2}=\left\langle\left(M^{\prime}\right)_{\tau, 0}^{+} M_{\tau, 0}^{-} \phi_{\tau}, \phi_{\tau}\right\rangle+\left\langle\left(M^{\prime}\right)_{\tau, 0}^{+}\left(I-M_{\tau, 0}^{-}\right) \phi_{\tau}, \phi_{\tau}\right\rangle+\left\|\phi_{\tau}\right\|^{2}= \\
\left\|M_{\tau, 0}^{-} \phi_{\tau}\right\|^{2}+\left\langle\left(M^{\prime}\right)_{\tau, 0}^{+}\left(I-M_{\tau, 0}^{-}\right) \phi_{\tau}, \phi_{\tau}\right\rangle+\left\|\phi_{\tau}\right\|^{2} .
\end{array}
$$

Therefore, for every $\tau \in X(k-1)$,

$$
\begin{array}{r}
\left\|d_{\tau} \phi_{\tau}\right\|^{2}-\frac{k}{k+1}\left\|\phi_{\tau}\right\|^{2}=\left\|d_{\tau} \phi_{\tau}\right\|^{2}-\left\|\phi_{\tau}\right\|^{2}+\frac{1}{k+1}\left\|\phi_{\tau}\right\|^{2}= \\
\left\|M_{\tau, 0}^{-} \phi_{\tau}\right\|^{2}+\left\langle\left(M^{\prime}\right)_{\tau, 0}^{+}\left(I-M_{\tau, 0}^{-}\right) \phi_{\tau}, \phi_{\tau}\right\rangle+\frac{1}{k+1}\left\|\phi_{\tau}\right\|^{2} .
\end{array}
$$


Also,

$$
\begin{aligned}
\left\|M_{\tau, 0}^{-} \phi_{\tau}\right\|^{2}= & \sum_{v \in X_{\tau}^{(0)}} m_{\tau}(\{v\})\left(\sum_{u \in X_{\tau}^{(0)}} \frac{m_{\tau}(\{u\})}{m_{\tau}(\emptyset)} \phi_{\tau}(\{u\})\right)^{2}= \\
& m_{\tau}(\emptyset)\left(\sum_{u \in X_{\tau}^{(0)}} \frac{m_{\tau}(\{u\})}{m_{\tau}(\emptyset)} \phi_{\tau}(\{u\})\right)^{2}=\left\|d_{\tau}^{*} \phi_{\tau}\right\|^{2} .
\end{aligned}
$$

Combining this with the previous inequality yields that

$$
\left\|d_{\tau} \phi_{\tau}\right\|^{2}-\frac{k}{k+1}\left\|\phi_{\tau}\right\|^{2}=\left\|d_{\tau}^{*} \phi_{\tau}\right\|^{2}+\left\langle\left(M^{\prime}\right)_{\tau, 0}^{+}\left(I-M_{\tau, 0}^{-}\right) \phi_{\tau}, \phi_{\tau}\right\rangle+\frac{1}{k+1}\left\|\phi_{\tau}\right\|^{2} .
$$

By Proposition 12

$$
\|d \phi\|^{2}=\sum_{\tau \in X(k-1)}\left(\left\|d_{\tau} \phi_{\tau}\right\|^{2}-\frac{k}{k+1}\left\|\phi_{\tau}\right\|^{2}\right)
$$

therefore

$$
\|d \phi\|^{2}=\sum_{\tau \in X(k-1)}\left(\left\|d_{\tau}^{*} \phi_{\tau}\right\|^{2}+\left\langle\left(M^{\prime}\right)_{\tau, 0}^{+}\left(I-M_{\tau, 0}^{-}\right) \phi_{\tau}, \phi_{\tau}\right\rangle+\frac{1}{k+1}\left\|\phi_{\tau}\right\|^{2}\right) .
$$

Using the equalities proven in Proposition 12, we deduce that

$$
\|d \phi\|^{2}=\left\|d^{*} \phi\right\|^{2}+\|\phi\|^{2}+\sum_{\tau \in X(k-1)}\left\langle\left(M^{\prime}\right)_{\tau, 0}^{+}\left(I-M_{\tau, 0}^{-}\right) \phi_{\tau}, \phi_{\tau}\right\rangle,
$$

as needed.

\section{Proof of Lemma 14:}

Proof. Let $\phi$ be as above. Recall that for every $\tau \in X(k-1), \phi_{\tau}$ decomposes orthogonally as

$$
\phi_{\tau}=\left(I-M_{\tau, 0}^{-}\right) \phi_{\tau}+M_{\tau, 0}^{-} \phi_{\tau},
$$

Therefore

$$
\begin{array}{r}
\left\langle\left(M^{\prime}\right)_{\tau, 0}^{+}\left(I-M_{\tau, 0}^{-}\right) \phi_{\tau}, \phi_{\tau}\right\rangle= \\
\left\langle\left(M^{\prime}\right)_{\tau, 0}^{+}\left(I-M_{\tau, 0}^{-}\right) \phi_{\tau},\left(I-M_{\tau, 0}^{-}\right) \phi_{\tau}\right\rangle+\left\langle\left(M^{\prime}\right)_{\tau, 0}^{+}\left(I-M_{\tau, 0}^{-}\right) \phi_{\tau}, M_{\tau, 0}^{-} \phi_{\tau}\right\rangle .
\end{array}
$$

As explained above, $\left(M^{\prime}\right)_{\tau, 0}^{+}\left(I-M_{\tau, 0}^{-}\right) \phi_{\tau} \in \operatorname{Im}\left(I-M_{\tau, 0}^{-}\right)$and therefore

$$
\left\langle\left(M^{\prime}\right)_{\tau, 0}^{+}\left(I-M_{\tau, 0}^{-}\right) \phi_{\tau}, M_{\tau, 0}^{-} \phi_{\tau}\right\rangle=0 .
$$

This yields that

$$
\left\langle\left(M^{\prime}\right)_{\tau, 0}^{+}\left(I-M_{\tau, 0}^{-}\right) \phi_{\tau}, \phi_{\tau}\right\rangle=\left\langle\left(M^{\prime}\right)_{\tau, 0}^{+}\left(I-M_{\tau, 0}^{-}\right) \phi_{\tau},\left(I-M_{\tau, 0}^{-}\right) \phi_{\tau}\right\rangle .
$$

Note that by definitions of $\mu_{k}, \nu_{k}$

$$
\left\langle\left(M^{\prime}\right)_{\tau, 0}^{+}\left(I-M_{\tau, 0}^{-}\right) \phi_{\tau},\left(I-M_{\tau, 0}^{-}\right) \phi_{\tau}\right\rangle \leq \mu_{k}\left\|\left(I-M_{\tau, 0}^{-}\right) \phi_{\tau}\right\|^{2} \leq \mu_{k}\left\|\phi_{\tau}\right\|^{2},
$$


and

$$
\left\langle\left(M^{\prime}\right)_{\tau, 0}^{+}\left(I-M_{\tau, 0}^{-}\right) \phi_{\tau},\left(I-M_{\tau, 0}^{-}\right) \phi_{\tau}\right\rangle \geq \nu_{k}\left\|\left(I-M_{\tau, 0}^{-}\right) \phi_{\tau}\right\|^{2} \geq \nu_{k}\left\|\phi_{\tau}\right\|^{2} .
$$

Summing over all $\tau \in X(k-1)$ and applying Proposition 12 yields the needed results, i.e.,

$$
\begin{array}{r}
\sum_{\tau \in X(k-1)}\left\langle\left(M^{\prime}\right)_{\tau, 0}^{+}\left(I-M_{\tau, 0}^{-}\right) \phi_{\tau}, \phi_{\tau}\right\rangle= \\
\sum_{\tau \in X(k-1)}\left\langle\left(M^{\prime}\right)_{\tau, 0}^{+}\left(I-M_{\tau, 0}^{-}\right) \phi_{\tau},\left(I-M_{\tau, 0}^{-}\right) \phi_{\tau}\right\rangle \leq \sum_{\tau \in X(k-1)} \mu_{k}\left\|\phi_{\tau}\right\|^{2}=(k+1) \mu_{k}\|\phi\|^{2},
\end{array}
$$

and a similar computation yields the second inequality of the Lemma. 